\title{
Non-Intrusive Detection of Combustion Intermediates by Photoionization via Rydberg States and Microwave Backscattering
}

\author{
Fedor Rudakov ${ }^{\mathrm{a}, \mathrm{b}, *}$, Yan Gao ${ }^{\mathrm{b}}$, Xinxin Cheng ${ }^{\mathrm{b}}$, Peter M. Weber ${ }^{\mathrm{b}}$ \\ ${ }^{a}$ Chemistry Department, University of Missouri, Kansas City, MO, 64110, USA \\ ${ }^{\mathrm{b}}$ Department of Chemistry, Brown University, Providence, RI, 02912, USA \\ *rudakovf@umkc.edu
}

\begin{abstract}
A non-intrusive, in-situ detection technique for combustion intermediates in flame environments yields highly resolved spectra that are largely insensitive to molecular vibrations and thus temperature. The technique is based on laser photoionization of target compounds via Rydberg states, followed by detection of the laser-induced plasma with microwave radiation. The feasibility of this approach is tested on methyl radicals that are detected in methane, propane, and hexane fuel-rich flames. The methyl radicals are prepared in the 3s Rydberg state using photoexcitation at $216.4 \mathrm{~nm}$. Using tunable VIS/IR radiation, the molecules are promoted from $3 \mathrm{~s}$ to the $3 p_{x y}$ and $3 p_{z}$ Rydberg states. Photoionization out of the $3 p$ states with $355 \mathrm{~nm}$ photons results in a small-volume plasma that is detected by microwave backscattering. The previously unobserved 3pxy state is found to have a binding energy of $2.57 \mathrm{eV}$.
\end{abstract}

Keywords: Laser spectroscopy, photoionization, combustion, methyl radical, CH3, RadarREMPI. 


\section{Introduction}

Understanding the mechanisms of combustion reactions is of great importance for basic combustion science as well as for the development of efficient and clean engines ${ }^{1}$. Knowledge of the distribution of the combustion intermediates within a flame provides valuable insights into kinetic parameters and reaction mechanisms. The concentrations of combustion intermediates in flames are often determined using VUV photoionization mass spectrometry ${ }^{2,3,4,5,6}$. Using tunable far-UV radiation sources, it is possible to measure both the ionization potential and the mass of combustion intermediates, thus allowing for isomer-specific detection. While it is possible to map the concentrations of transients across a flame, photoionization mass spectrometry requires the extraction of combustion intermediates from the flame. The sampling cone perturbs the temperature and flow fields of the flame. Therefore, photoionization mass spectrometry may provide inaccurate results. Non-intrusive and in-situ detection is usually achieved by probing vibrational spectra using cavity ring-down spectroscopy (CRDS), coherent anti-Stokes Raman scattering (CARS), or degenerate four-wave mixing (DFWM) [See Ref. 7 and references within]. However, those techniques are often challenged when one seeks detection of medium to largesized combustion intermediates in flames, since the complexity of vibrational spectra scales very unfavorably with both molecular size and the amount of vibrational energy available to the molecule. Laser-induced fluorescence (LID) has also limited applicability because fluorescence spectra are often broad and not characteristic and because many combustion intermediates do not fluoresce.

We propose here a technique for detection of combustion intermediates that utilizes resonance enhanced multiphoton ionization (REMPI) via molecular Rydberg states. Molecular Rydberg 
states are excited electronic states with wavefunctions resembling those of a hydrogen atom. Unlike valence electrons, Rydberg electrons do not contribute significantly to molecular bonding, so that the geometrical structures, and potential energy surfaces, of molecules in Rydberg states are nearly identical to those of the corresponding ion states ${ }^{8}$. Since the potential energy surfaces of all the Rydberg states and the corresponding ion state are nearly identical, the same is true for vibrational wavefunctions of all Rydberg states and an ion state.

This results in several unique properties: First, most of the Frank-Condon envelope for RydbergRydberg or Rydberg-ion transitions is enclosed in a very narrow band [no vibrational quantum number change is allowed $(\Delta v=0)]$. Therefore, transitions between two Rydberg states or between a Rydberg state and an ion state reveal highly resolved spectra ${ }^{9,10,11}$. This also implies that Rydberg spectra are insensitive towards vibrational motion that is, they are not sensitive to temperature. Secondly, since the number of molecular Rydberg states mirrors those of atoms, the complexity of Rydberg spectra does not scale with the molecular size, making the technique well suited for the detection of large molecules. ${ }^{12}$ Finally, Rydberg orbitals extend across an entire molecule, thus providing sensitivity to the global structure of the molecule. Yet Rydberg spectra remain very sensitive to even minor changes in the molecular structure, including conformeric shapes. ${ }^{13,14,15,16}$ Because the method can be implemented with ultrafast time resolution, it is also possible to apply the photoionization via Rydberg states to probe molecular structure and dynamics in highly energetic molecules. ${ }^{17,18,19,20,21}$ The combination of high sensitivity to the molecular structure, insensitivity to vibrational motion, and applicability to large molecules makes it attractive to suggest photoionization through Rydberg states as a tool for detection of combustion intermediates. 
We note that the insensitivity of Rydberg spectra to vibrational motion is based on an adiabatic approximation. If Rydberg states are short-lived or heavily mixed with valence states, the Rydberg-Rydberg transitions can be encumbered by vibrational motion. Furthermore, since the lifetimes of the excited electronic states typically depend on the amount of vibrational energy available to the molecule, the temperature of the molecules in a flame may affect the intensities of the Rydberg peaks and their widths.

Molecular Rydberg spectra are usually observed by photoionization coupled with mass spectrometry or photoelectron spectroscopy. When applied to combustion environments, both techniques would require extraction of combustion intermediates from the flame, thus preventing non-intrusive and in-situ detection. Recently we demonstrated that molecular Rydberg spectra could be acquired via microwave scattering from plasma produced by photoionization. ${ }^{22,23}$. Detection is initiated by a microwave beam that is directed toward the ionization region ${ }^{24}$. The electrons generated through resonance-enhanced multiphoton ionization (REMPI) create a weakly ionized, small-volume plasma that scatters microwave radiation. The intensity of the scattering signal reflects the generation and evolution of the unbounded electrons inside the plasma. As the photoionization laser wavelength is tuned through the resonances associated with Rydberg-Rydberg transitions, the total electron number in the plasma (and thus the intensity of scattered microwaves) changes, thereby revealing the Rydberg spectrum.

For a proof-of-a-feasibility demonstration we applied the photoionization via Rydberg states followed by detection of the laser-induced plasma to the detection of methyl radicals in methane, propane, and hexane flames. For methane and propane flames, we also performed spatially resolved measurements. The spectra show sharp peaks corresponding to transitions from $3 \mathrm{~s}$ to $3 p_{z}, 3 p_{x y}$, as well as higher-lying Rydberg states of the methyl radicals. We note here that the 
$3 p_{\mathrm{xy}}$ Rydberg state in methyl radicals, while predicted computationally, ${ }^{25,26}$ appears not to have been previously observed experimentally. REMPI $^{27,28,29,30}$ as well as other techniques ${ }^{31,32}$ have been applied to detection of methyl radicals. Those measurements were performed in methane flames, which represent a relatively straightforward environment that delivers high concentrations of methyl radicals. The photoionization through Rydberg states affords a high degree of selectivity that allows us to detect methyl radicals in more complex environments such as propane and hexane flames. We anticipate that the technique can be utilized for detection of polyatomic combustion intermediates in complex environments.

\section{Experimental Setup}

Our experimental setup consists of two OPGs pumped by a $10 \mathrm{~Hz}, 21 \mathrm{ps}, 70 \mathrm{~mJ}$ Nd:YAG laser (Ekspla PL2251C) and a microwave homodyne transceiver system (figure 1a). The harmonics generation produces three beams with wavelengths of $355 \mathrm{~nm}, 532 \mathrm{~nm}$, and $1064 \mathrm{~nm}$. The 355 $\mathrm{nm}$ and $532 \mathrm{~nm}$ beams are used to pump two optical parametric generators. The $1064 \mathrm{~nm}$ output is upconverted to produce $355 \mathrm{~nm}$ pulses. We will refer to the $532 \mathrm{~nm}$ pumped optical parametric generator (Ekspla PG501-DFG1) as OPG1. The $355 \mathrm{~nm}$ pumped optical parametric generator (Ekspla PG403A-SH), which is equipped with a second harmonic generation unit, will be referred to OPG2. The outputs from both OPGs and the $355 \mathrm{~nm}$ beam are overlapped in space (using a set of dichroic mirrors) and in time (using optical delay stages not shown in figure 1a). The beams, containing photons of three different wavelengths, are focused by a lens ( $\mathrm{f}=250 \mathrm{~mm}$ ) into the flame creating a plasma that is $100 \mu \mathrm{m}$ in diameter at the beam waist and $60 \mathrm{~mm}$ long. Our optical layout also included a periscope, installed on a motorized vertical linear stage, to adjust the position of the laser beams with respect to the surface of the burner. The $355 \mathrm{~nm}$ 
pulses have energies of $1.6 \mathrm{~mJ}$ and a linewidth of $\sim 1 \mathrm{~cm}^{-1}$. The tuning curves for the OPGs are shown in figure $1 \mathrm{~b}$. All energies were measured at the target. The laser power densities produced by the OPGs at the beam waist are $\sim 3 \cdot 10^{10} \mathrm{~W} / \mathrm{cm}^{2}$ and the power density produced by the 355 $\mathrm{nm}$ pulse is $\sim 2 \cdot 10^{12} \mathrm{~W} / \mathrm{cm}^{2}$. The durations of all laser pulses are about $10-12 \mathrm{ps}$. The line widths of laser pulses produced by OPG1 and OPG2 are $\sim 6 \mathrm{~cm}^{-1}$, and $\sim 16 \mathrm{~cm}^{-1}$ respectively.

Focusing the laser beam containing pulses of three different wavelengths overlapped in time results in photoionization of methyl radicals according to the scheme shown in figure 1c. The molecules are excited by $216.4 \mathrm{~nm}$ photons (produced by OPG2) into the 3s Rydberg state, and ionized out of that state by VIS/IR photons (produced by OPG1) and $355 \mathrm{~nm}$ photons. Based on the methyl radical ionization potential of $9.84 \mathrm{eV}^{33}$ and the photoexcitation energy of the $3 \mathrm{~s}$ state of $5.73 \mathrm{eV}^{34}$, we estimate the binding energy of the 3s Rydberg state as $4.11 \mathrm{eV}$. Therefore, the $\mathrm{CH}_{3}$ radical cannot be ionized out of the $3 \mathrm{~s}$ Rydberg state using a single $355 \mathrm{~nm}$ photon (3.49 $\mathrm{eV}$ ). The binding energies of the $3 \mathrm{p}_{\mathrm{z}}$ Rydberg state is $2.41 \mathrm{eV}$ (calculated based on the photoexcitation energy of $7.44 \mathrm{eV}^{35,36}$ ). The binding energy of the $3 p_{\mathrm{xy}}$ state is $2.57 \mathrm{eV}$ (as discussed in the Appendix). $\mathrm{CH}_{3}$ radicals can readily be ionized out of $3 p$ states using a single $355 \mathrm{~nm}$ photon. As the wavelength of the photons produced by OPG1 is tuned through the resonances associated with $3 \mathrm{~s} \rightarrow 3 \mathrm{p}$ Rydberg transitions, the ionization efficiency strongly increases at the resonance conditions.

Photoionization of combustion intermediates results in a small-volume plasma that is probed with a microwave homodyne transceiver detection system that has been described previouslyError! Bookmark not defined.. Briefly, an output of a $20 \mathrm{dBm}, 10 \mathrm{GHz}$ microwave source is split into two parts. One part is used to illuminate the laser-induced plasma through a microwave horn while the second part is used for the homodyne mixer. Microwave 
backscattering from the plasma is collected by the same microwave horn and is separated from the transmitted microwaves by a circulator. The received signal is amplified and mixed with the source. The microwave at the difference frequency is amplified again and recorded by a digitizer that is triggered by the laser.

The experiments were conducted in a flat, fuel-rich flame produced by a McKenna Burner (Holthuis \& Associates). The fuel was premixed with an $\mathrm{Ar} / \mathrm{O}_{2} 80 / 20$ mixture to a 1.64 equivalence ratio. The flow of $\mathrm{Ar} / \mathrm{O}_{2}$ in all measurements was set at $14.0 \mathrm{slpm}$ (standard liters per minute). Methane flow was $2.30 \mathrm{slpm}$, propane flow was $0.92 \mathrm{slpm}$, and hexane flow was $2.83 \mathrm{ml} / \mathrm{min}$. The flow of gases was controlled using mass flow controllers. The flow of hexane was controlled using a syringe pump.

\section{Results and Discussion}

Three-color photoionization of methane, propane, and hexane flames (Fig. 1c) results in spectra shown in figure 2. All spectra were acquired $\sim 0.5$ to $0.7 \mathrm{~mm}$ from the surface of the burner. The average of two scans is shown. All spectra feature two sharp peaks centered at $807 \mathrm{~nm}$ and 728.5 nm. We thus can conclude that both peaks originate from a combustion intermediate that is common to all three flames. As will be shown below, both peaks originate from photoionization of methyl radicals. The peaks are observed on top of a fairly strong background that likely originates from photoionization of other flame components with UV pulses (216.4 nm and 355 $\mathrm{nm}$ ). As expected, the strongest background (relative to the intensity of the peaks) was observed for hexane flame ( $\sim 90 \%$ of the maximum microwave scattering intensity) while the methane flame features the smallest background relative to the maximum intensity $(\sim 50 \%)$. Indeed, 
combustion of polyatomic hydrocarbons results in a large variety of complex combustion intermediates 6 that can be readily ionized by UV photons along with methyl radicals.

In order to demonstrate that all the peaks in figure 2 originate from methyl radicals we tuned the pump photon energy in the range from $210 \mathrm{~nm}$ to $220 \mathrm{~nm}$ and recorded three photoionization spectra of the methyl flame (Fig. 3, top). In one measurement (which serves as a background), photoionization was performed with $210-220 \mathrm{~nm}$ and $355 \mathrm{~nm}$ photons only (black line). In two other measurements in addition to $210-220 \mathrm{~nm}$ and $355 \mathrm{~nm}$ pulses we also introduced VIS/IR pulses at $728.5 \mathrm{~nm}$ (blue line), and $807 \mathrm{~nm}$ (red line) produced by OPG1. The difference spectra are shown in the bottom of figure 3. An intense feature centered near $216.4 \mathrm{~nm}$ that appears in all spectra acquired with VIS/IR photons perfectly matches (within the bandwidth of our laser) the well-known transition energy between the ground state and the $3 \mathrm{~s} \operatorname{state}^{33}$. We thus attribute both peaks in figure 2 to photoionization of methyl radical out of the 3s Rydberg state.

The difference in energy between the $3 \mathrm{~s}$ and $3 \mathrm{p}_{\mathrm{z}}$ Rydberg state in $\mathrm{CH}_{3}$ radical is $13733 \mathrm{~cm}^{-1}$, which corresponds to $728.2 \mathrm{~nm}^{33}$. This number matches (within the bandwidth of our laser) the most intense peak in the spectrum centered at $728.5 \mathrm{~nm}^{33}$. We thus can assign the $728.5 \mathrm{~nm}$ peak as the $3 p_{z}$ Rydberg state in methyl radical. The peak centered at $807 \mathrm{~nm}$ can be attributed to the transition to the $3 p_{x y}$ state of the methyl radical. While this $3 p_{x y}$ state has not been previously experimentally observed, we support this assignment with quantum-chemical calculations (see appendix).

We note that all three spectra show a series of sharp peaks (the most intense centered at 217.6 $\mathrm{nm})$. Those peaks may be attributed to electronic transitions in atoms. The spectral resolution is limited by the bandwidth of our laser $(\sim 0.08 \mathrm{~nm}$ at $217.6 \mathrm{~nm})$ and the step size of $0.1 \mathrm{~nm}$. In order to identify the atoms, a higher spectral resolution would be required. 
Figures $4 \mathrm{a}$ and $4 \mathrm{~b}$ show the position-dependent spectra of methane and propane flames respectively. Both spectra were obtained using the photoionization scheme shown in figure 1c. The insets show the intensities of the $3 p_{z}$ and $3 p_{x y}$ states as a function of distance from the burner (the background caused by photoionization of other combustion intermediates is subtracted). The intensities of the $3 p$ states of methyl radical in methane flame reaches the maximum near $1 \mathrm{~mm}$ from the surface of the burner, which is in agreement with literature values ${ }^{29,30}$. The concentration of methyl radical in propane flame peaks at $\sim 0.65 \mathrm{~mm}$. Combustion of propane has been studied before ${ }^{37}$. However, those measurements were performed under low pressure and cannot be used for comparison.

We note that intensities of the $3 p_{z}$ and $3 p_{x y}$ peaks show slightly different dependencies on the position of the laser focus with respect to the surface of the burner. There can be several explanations for this. First, microwave scattering provides a non-linear response with photoionization efficiency. Secondly, it might be an artifact of the background subtraction: we assumed that the background caused by photoionization of combustion intermediates other than $\mathrm{CH}_{3}$ radical is changing gradually and utilized a linear interpolation to estimate it. Finally, the lifetimes of the excited electronic states are dependent on the temperature ${ }^{23}$. In fact, the lifetimes of the $3 p_{z}$ and $3 p_{x y}$ states may be disproportionally affected by the temperature. We mention here that while the relative peak intensities are affected by the temperature the peak positions are largely unaffected by vibrational motion.

For the reasons listed above, the intensities of the $3 p_{z}$ and $3 p_{x y}$ peaks do not exactly reflect the relative concentration of $\mathrm{CH}_{3}$ in the flame radical. Nevertheless, as has been previously noted, a calibration of the technique is feasible in principle ${ }^{29,30}$. To achieve an accurate calibration, the dependence of the lifetime of the excited electronic states on the temperature should be taken 
into account. Alternatively, the dependence of photoionization efficiency on the temperature can be eliminated by utilizing ultrashort laser pulses.

\section{Conclusion}

In summary, we present a technique for detection of combustion intermediates that relies on the unique properties of molecular Rydberg states to reveal highly resolved and very characteristic spectra that are largely insensitive to vibrational motion (i.e., temperature). The technique delivers a very high degree of selectivity, allowing us to detect combustion intermediates in complex environments such as hexane flames. The selectivity of the technique might be further enhanced by utilizing femtosecond laser pulses and introducing a time delay between excitation and ionization pulses. In that way, discrimination between various combustion intermediates could also be achieved based on the lifetimes of the excited electronic states.

We note that a quantitative calibration of the technique is feasible but needs to address the nonlinear response of the microwave scattering signal and the dependence of the lifetimes of the excited electronic states on temperature. We also note that structure-sensitive photoionization through Rydberg states could be implemented in combination with mass spectrometry. A tandem of mass spectrometry and photoionization through Rydberg states is expected to deliver a highly resolved, two-dimensional spectrum that is insensitive towards molecular vibrations. RydbergRydberg transitions can also be probed via non-linear spectroscopic techniques such as degenerate four-wave mixing and coherent anti-Stokes Raman spectroscopy. Since the complexity of the Rydberg spectrum does not scale with the molecular size we anticipate that the 
technique can be utilized for detection of polyatomic combustion intermediates. Research in this direction is currently ongoing in our laboratory.

\section{Acknowledgement}

The authors acknowledge support by the National Science Foundation, Grant No. CBET1336105.

\section{Appendix}

To substantiate the assignment of the observed $807 \mathrm{~nm}$ peak to the transition from $3 \mathrm{~s}$ to $3 \mathrm{p}_{\mathrm{xy}}$, we obtained the structure of the methyl radical in the ion state by the DFT-B3LYP method with an AUG-cc-pVTZ basis set in Gaussian $09^{38}$ and performed Perdew-Zunger self-interactioncorrected density functional theory (PZ-SIC) calculations. The calculations were implemented in the GPAW program in real space with a uniform grid and the local density approximation (LDA) functional ${ }^{39}$ by using a procedure described in detail previously ${ }^{40,41}$. In the PZ-SIC calculations, the side length of the cubic simulation cell was set as $30 \AA$ with a uniform grid size of $0.15 \AA$. The orbital energies of the singly occupied molecular orbitals were used to estimate the binding

energies of the Rydberg states ${ }^{42}$. The results for the $3 \mathrm{~s}, 3 \mathrm{p}_{\mathrm{x}, \mathrm{y}}$, and $3 \mathrm{p}_{\mathrm{z}}$ Rydberg states are 4.12 , 2.66 , and $2.48 \mathrm{eV}$, respectively.

For the 3s Rydberg state, the calculated value of $4.12 \mathrm{eV}$ agrees very well with the value of 4.11 $\mathrm{eV}$ measured here, which is obtained by subtracting the excitation laser photon energy, $5.73 \mathrm{eV}$, from the well-known ionization energy of $9.84 \mathrm{eV}^{33}$.

For $3 \mathrm{p}_{\mathrm{z}}$, the PZ-SIC calculation gives a binding energy of $2.48 \mathrm{eV}$. With a calculated $3 \mathrm{~s}$ binding energy of $4.12 \mathrm{eV}$, this places the energy for the $3 \mathrm{~s} \rightarrow 3 \mathrm{p}_{\mathrm{z}}$ transition at $1.64 \mathrm{eV}$. Experimentally, the $3 \mathrm{~s} \rightarrow 3 \mathrm{p}_{\mathrm{z}}$ resonance transition is found at $728.5 \mathrm{~nm}$, or $1.70 \mathrm{eV}$. This suggests that the PZ- 
SIC calculation is low by $0.06 \mathrm{eV}$, well within the stated accuracy of the computational technique of $0.1 \mathrm{eV}^{40}$. These values, as well as literature values, are listed in Table 1.

For $3 p_{x y}$ the PZ-SIC calculation gives a binding energy of $2.66 \mathrm{eV}$. Given the $4.12 \mathrm{eV}$ binding energy of $3 \mathrm{~s}$, the computation therefore predicts a transition energy of $1.46 \mathrm{eV}$. This seems to be a good match to the observed peak at $807 \mathrm{~nm}=1.54 \mathrm{eV}$. With this assignment, the computed transition energy is low by $0.08 \mathrm{eV}$, which is close to the deviation found for the well-established $3 p_{z}$ state, see table 1 . We therefore suggest that the experimentally observed spectral line at 807 $\mathrm{nm}$ be assigned to the $3 \mathrm{~s} \rightarrow 3 \mathrm{p}_{\mathrm{xy}}$ transition. This assignment places the experimental binding energy of the $3 p_{x y}$ state at $4.11 \mathrm{eV}-1.54 \mathrm{eV}=2.57 \mathrm{eV}$.

Table 1: Transition energies between Rydberg states, including the proposed assignment of the $3 p_{x y}$ Rydberg state. All energies are in $\mathrm{eV}$.

\begin{tabular}{cccccc}
\hline \hline $\begin{array}{c}\text { Rydberg } \\
\text { transition }\end{array}$ & $\begin{array}{c}\text { Prior } \\
\text { computational } \\
\text { work }\end{array}$ & $\begin{array}{c}\text { Prior } \\
\text { experimental } \\
\text { work }\end{array}$ & $\begin{array}{c}\text { Present } \\
\text { Experiment }\end{array}$ & $\begin{array}{c}\text { Present } \\
\text { computation } \\
\text { (PZ-SIC) }\end{array}$ & $\begin{array}{c}\text { Difference } \\
\text { (present } \\
\text { experiment }- \\
\text { computation) }\end{array}$ \\
\hline $3 \mathrm{~s} \rightarrow 3 \mathrm{p}_{\mathrm{xy}}$ & $1.03^{(25)}, 1.24^{(26)}$ & -- & 1.54 & 1.46 & 0.08 \\
$3 \mathrm{~s} \rightarrow 3 \mathrm{p}_{\mathrm{z}}$ & $1.81^{(25)}, 1.51^{(26)}$ & $1.70^{(33)}$ & 1.70 & 1.64 & 0.06 \\
\hline
\end{tabular}

\section{Figure Captions}

Figure 1. (color online) Schematic diagram of the experimental setup (a) and photoionization scheme (c). The $70 \mathrm{~mJ}, 21 \mathrm{ps}, 10 \mathrm{~Hz}$ output of the Nd:YAG laser is unconverted to produce three beams at $1064 \mathrm{~nm}, 532 \mathrm{~nm}$, and $355 \mathrm{~nm}$. The $355 \mathrm{~nm}$-pumped OPG features a second harmonic generator unit and is tuned to produce $216.4 \mathrm{~nm}$ pulses that prepare the molecule in the $3 \mathrm{~s}$ Rydberg state. The $532 \mathrm{~nm}$-pumped OPG produces tunable radiation that is used to promote the molecules from the $3 \mathrm{~s}$ to the $3 p$ Rydberg states. Out of those states, the molecule is ionized via 
$355 \mathrm{~nm}$ photons that are produced by upconversion of $1064 \mathrm{~nm}$ photons using LBO crystals. The OPG outputs and the $355 \mathrm{~nm}$ pulses are overlapped in time using linear delay stages (not shown). The tuning curves for the two OPGs are shown in (b).

Figure 2 (color online) Microwave backscattering intensity for methane, propane, and hexane flames as a function of VIS/IR photon wavelength. The photoionization was performed using $216.4 \mathrm{~nm}, 355 \mathrm{~nm}$, and VIS/IR photons produced by OPG1. The intensity of microwave scattering as a function of VIS/IR photon wavelength is recorded.

Figure 3 (color online) Microwave scattering intensity as a function of pump photon wavelength. Top: The black line represents the photoionization spectrum with $210-220 \mathrm{~nm}$ and $355 \mathrm{~nm}$ photons only and serves as a background. Introducing 728.5 and $807 \mathrm{~nm}$ pulses results in spectra represented by red and blue lines respectively. Bottom: Same spectra with the background subtracted.

Figure 4 (color online) Microwave scattering as a function of the VIS/IR photon wavelength and the distance from the surface of the burner for a) a methane flame and b) a propane flame. The insets show the position-dependent intensities of the 3pz and 3pxy states (the background caused by photoionization of combustion intermediates other than methyl radical is subtracted).

\section{References}

1 J.A. Miller, M.J. Pilling, and E. Troe, "Unraveling combustion mechanisms through a quantitative understanding of elementary reactions" Proc. Combust. Inst. 30 (2005) 43-48.

${ }^{2}$ N. Hansen, S.J. Klippenstein, J.A. Miller, J. Wang, T.A. Cool, M.E. Law, P.R. Westmoreland, T. Kasper, and K. Kohse-Höinghaus "Identification of $\mathrm{C}_{5} \mathrm{H}_{\mathrm{x}}$ isomers in fuel-rich flames by photoionization mass spectrometry and electronic structure calculations" J. Phys. Chem. A. 110 (2006) 4376-4388.

${ }^{3}$ C.A. Taatjes, CA, N. Hansen, A. McIlroy, J.A. Miller, J.P. Senosiain, S.J. Klippenstein, F. Qi, L.S. Sheng, Y.W. Zhang, T.A. Cool, J. Wang, P.R. Westmoreland, M.E. Law, ME, T. Kasper, 
and K. Kohse-Höinghaus "Enols are common intermediates in hydrocarbon oxidation" Science 308 (2005) 1887-1889.

${ }^{4}$ N. Hansen, J.A. Miller, P.R. Westmoreland, T. Kasper, K. Kohse-Höinghaus, J. Wang, and T.A. Cool, "Isomer-specific combustion chemistry in allene and propyne flames" Combust. Flame 156(11) (2009) 2153-2164.

5 Y. Li and F. Qi "Recent Applications of Synchrotron VUV Photoionization Mass Spectrometry: Insight into Combustion Chemistry" Acc. Chem. Res. 43(1) 2010, 68-78

${ }^{6}$ F. Qi “Combustion chemistry probed by synchrotron VUV photoionization mass spectrometry" P. Combust. Inst. 34(1) 2013 33-63

${ }^{7}$ S. Cheskis, A. Goldman "Laser diagnostics of trace species in low-pressure flat flame" Prog. Energy Combust. Sci. 35 (2009) 365-382.

${ }^{8}$ J. L. Gosselin and P.M. Weber "Rydberg Fingerprint Spectroscopy: A New Spectroscopic Tool With Local And Global Structural Sensitivity” J. Phys. Chem. A, 109 (2005) 4899 - 4904.

9 J.D. Cardoza and P.M. Weber, "Resolved: Electronic states underneath broad absorptions" J. Chem. Phys. 127(3) (2007) 036101.

${ }^{10}$ J.L. Gosselin, M.P. Minitti, F.M. Rudakov, T.I. Sølling and P.M. Weber, "Energy flow and fragmentation dynamics of N,N-dimethylisopropylamine” J. Phys. Chem. A 110 (2006) 4251

${ }^{11}$ J.D. Cardoza, F.M. Rudakov, and P.M. Weber "Electronic Spectroscopy and Ultrafast Energy Relaxation Pathways in the lowest Rydberg States of Trimethylamine," J. Phys. Chem. A, 112(43) 2008 10736-10743.

12 J. D. Cardoza, F. M. Rudakov, N. Hansen, P. M. Weber, "Identification of isomeric flame components by Rydberg ionization spectroscopy," J. Electron. Spectrosc. Relat. Phenom. 165 (2008) 5-10.

13 M.P. Minitti and P.M. Weber, "Time-resolved conformational dynamics in hydrocarbon chains" Phys. Rev. Lett., 98, (2007), 253004.

14 J. Bao and P.M. Weber "Ultrafast Dynamics of Highly Excited Trans-Stilbene: A Different Twist” J. Phys. Chem. Lett. 1 (2010) 224-227.

15 J.C. Bush, M.P. Minitti, and P,M. Weber. "Ultrafast Formation of an Intramolecular Cation-Pi Bond" J. Photochem. Photobiol. A 213 (2010) 70-72.

16 J.C. Bush, M.P. Minitti, and P.M. Weber, "Dissociative energy flow, vibrational energy redistribution and conformeric structural dynamics in bifunctional amine model systems," $J$. Phys. Chem. A 114, (2010) 11078-11084.

${ }^{17}$ S. Deb, B. Bayes, M.P. Minitti, and P.M. Weber, "Structural Dynamics in Floppy Systems: Ultrafast Conformeric Motions in Rydberg-Excited Triethylamine," J. Phys. Chem. A 115, (2011) 1804-1809.

${ }^{18}$ F. Rudakov and P.M. Weber, "Ultrafast structural and isomerization dynamics in the Rydbergexited Quadricyclane - Norbornadiene System” J. Chem. Phys. 136 (2012) 134303.

19 S. Deb, X. Cheng, and P.M. Weber, "Structural Dynamics and Charge Transfer in Electronically Excited N,N'-Dimethylpiperazine" J. Phys. Chem. Lett. 4, (2013) 2780-2784. 
${ }^{20}$ X. Cheng, Y. Zhang, S. Deb, M.P. Minitti, Y. Gao, H. Jónsson, and P.M. Weber, "Ultrafast Structural Dynamics in Rydberg Excited N,N,N',N'-Tetramethylethylenediamine: Conformation Dependent Electron Lone Pair Interaction and Charge Delocalization," Chem. Sci., 5(11) (2014) $4394-4403$

${ }^{21}$ X. Cheng, Y. Zhang, Y. Gao, H. Jońsson, and P.M. Weber, "Ultrafast Structural Pathway of Charge Transfer in N,N,N,N-Tetramethylethylenediamine," J. Phys. Chem. A, 119(12) (2015) 2813-2818.

22 F. Rudakov, Z. Zhang "Standoff detection of large organic molecules using Rydberg fingerprint spectroscopy and microwave Rayleigh scattering” Opt. Lett. 37(2) (2012) 145-147

${ }^{23}$ F. Rudakov, Y. Zhang, X. Cheng, and P.M. Weber, "Standoff Trace Chemical Sensing via Manipulation of Excited Electronic State Lifetimes" Opt. Lett. 38 (21) (2013) 4445-4448.

${ }^{24}$ Z. Zhang, M.N. Shneider, R.B. Miles, "Coherent Microwave Rayleigh scattering of Resonance Enhanced Multiphoton Ionization in Argon", Phys. Rev. Lett. 98 (2007) 265005.

${ }^{25}$ G. Balerdi, J. Woodhouse, A. Zanchet, R. de Nalda, M.L. Senent, A. García-Velab, and L. Bañares "Femtosecond predissociation dynamics of the methyl radical from the $3 \mathrm{pz}$ Rydberg state" Phys. Chem. Chem. Phys. 18 (2016) 110-118

${ }^{26}$ H. T. Yu, A. Sevin, E. Kassab and E. M. Evleth, "A comparative theoretical analysis of the photochemistry of the methyl radical and related systems" J. Chem. Phys. 80(5) (1984), 20492059.

${ }^{27}$ K.C. Smyth and P.H. Taylor "Detection of the methyl radical in a methane/air diffusion flame by multiphoton ionization spectroscopy" Chem. Phys. Lett. 122(5) (1985) 518-522.

${ }^{28}$ Y. Wu, A. Bottom, Z. Zhang, T.M. Ombrello, and V.R. Katta, "Direct Measurement of Methyl Radical in Methane/Air Flame at Atmospheric Pressure by Radar REMPI" Opt. Express 19(24) (2011) 23997-24004

29 Y. Wu, Z. Zhang, "Two Dimensional Quantitative Measurements of Methyl Radicals in Methane/Air Flame" Appl. Opt. 54(2) (2015) 157-162

${ }^{30}$ Y. Wu, Z. Zhang, T.M. Ombrello, and V.R. Katta, "Quantitative Radar REMPI Measurements of Methyl Radicals in Flames at Atmospheric Pressure" Appl. Phys. B 111 (2013) 391-397

${ }^{31}$ V. Sick, M.N. Bui-Pham, and R.L. Farrow "Detection of methyl radicals in a flat flame by degenerate four-wave mixing" Opt. Lett. 20(19) (1995) 2036-2038.

32 J. J. Scherer, K. W. Aniolek, N. P. Cernansky, and D. J. Rakestraw "Determination of methyl radical concentrations in a methane/air flame by infrared cavity ringdown laser absorption spectroscopy" J. Chem. Phys. 107 (1997) 6196-6203.

${ }^{33}$ NIST webbook http://webbook.nist.gov/

34 T.B. Setterstena, R.L. Farrow, J.A. Gray "Coherent infrared-ultraviolet double-resonance spectroscopy of $\mathrm{CH}_{3}$ " Chem. Phys. Lett. 370 (2003) 204-210.

35 J. Heinze, N. Heberle, K. Kohse-Höinghaus "The CH3 3pz2A2" $\leftarrow \tilde{X} 2 \mathrm{~A} 2$ " 000 band at temperatures up to $1700 \mathrm{~K}$ investigated by REMPI spectroscopy" Chem. Phys. Lett. 223(4) (1994) 305-312. 
36 J.W. Hudgens, T. G. DiGiuseppe, and M. C. Lin "Two photon resonance enhanced multiphoton ionization spectroscopy and state assignments of the methyl radical" $J$. Chem. Phys. 79 (1983) 571-582.

${ }^{37}$ T.A. Cool, K. Nakajima, C.A. Taatjes, A. McIlroy, P.R. Westmoreland, M.E. Law, A. Morel "Studies of a fuel-rich propane flame with photoionization mass spectrometry" P. Combust. Inst. 30 (2005) 1681-1688.

${ }^{38}$ M. J. Frisch, et al., Gaussian 09, Revision D.01, Gaussian, Inc., Wallingford CT, 2009.

${ }^{39}$ J. P. Perdew and Y. Wang, "Accurate and Simple Analytic Representation of the Electron-Gas Correlation-Energy" Phys. Rev. B: Condens. Matter Mater. Phys., (45)1992 13244-13249.

${ }^{40}$ H. Gudmundsdóttir, Y. Zhang, P.M. Weber, and H.s Jónsson, "Self-interaction corrected density functional calculations of molecular Rydberg states," J. Chem. Phys. 139 (2013) 194102.

${ }^{41}$ H. Gudmundsdóttir, Y. Zhang, P.M. Weber and H. Jónsson, "Self-interaction corrected density functional calculations of Rydberg states of molecular clusters: N,N-dimethylisopropylamine," $J$. Chem. Phys. 141 (2014) 234308.

${ }^{42}$ X. Cheng, Y. Gao, F. Rudakov and P. M. Weber, "Charge transfer and ultrafast nuclear motions: the complex structural dynamics of an electronically excited triamine," Chemical Science. 7 (2016) 619. 


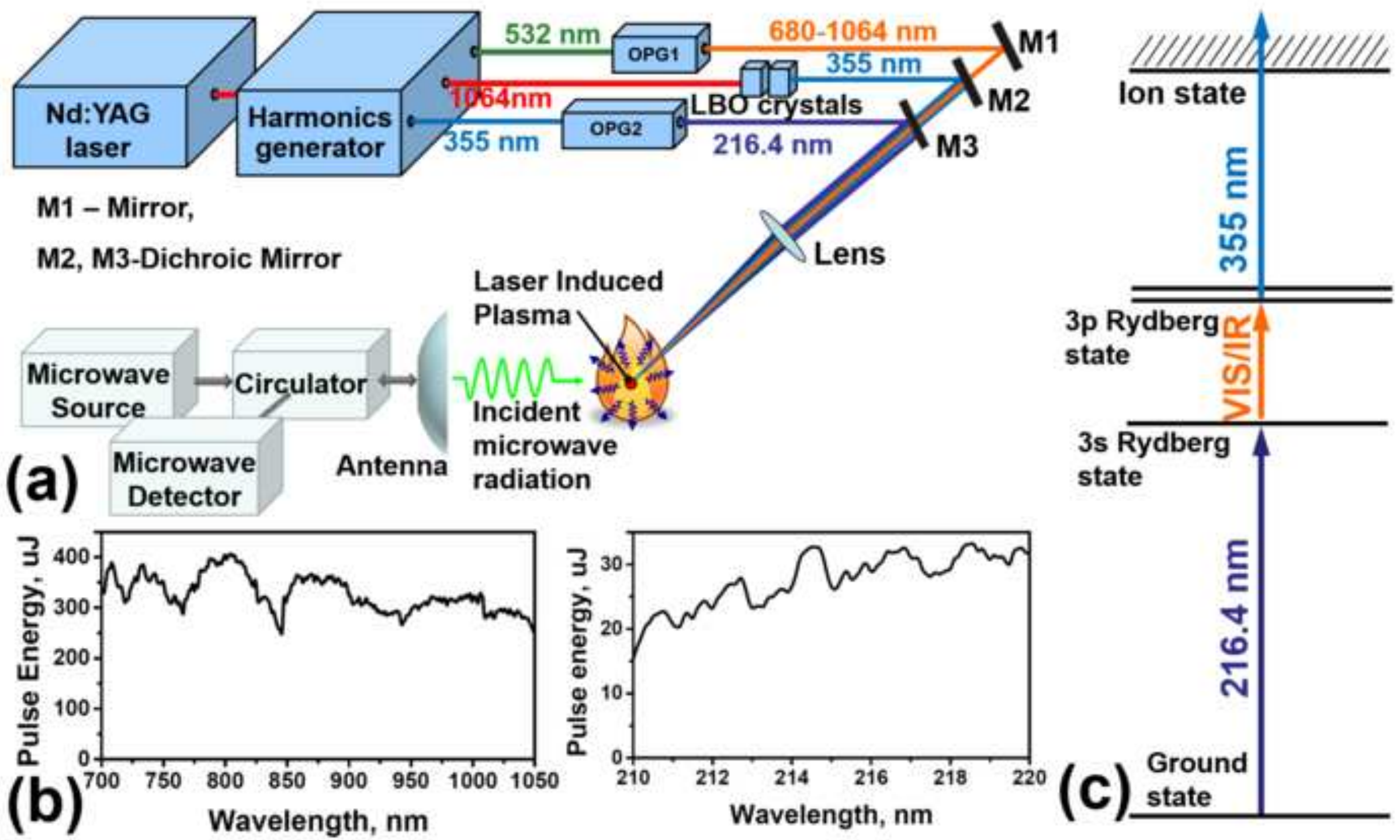




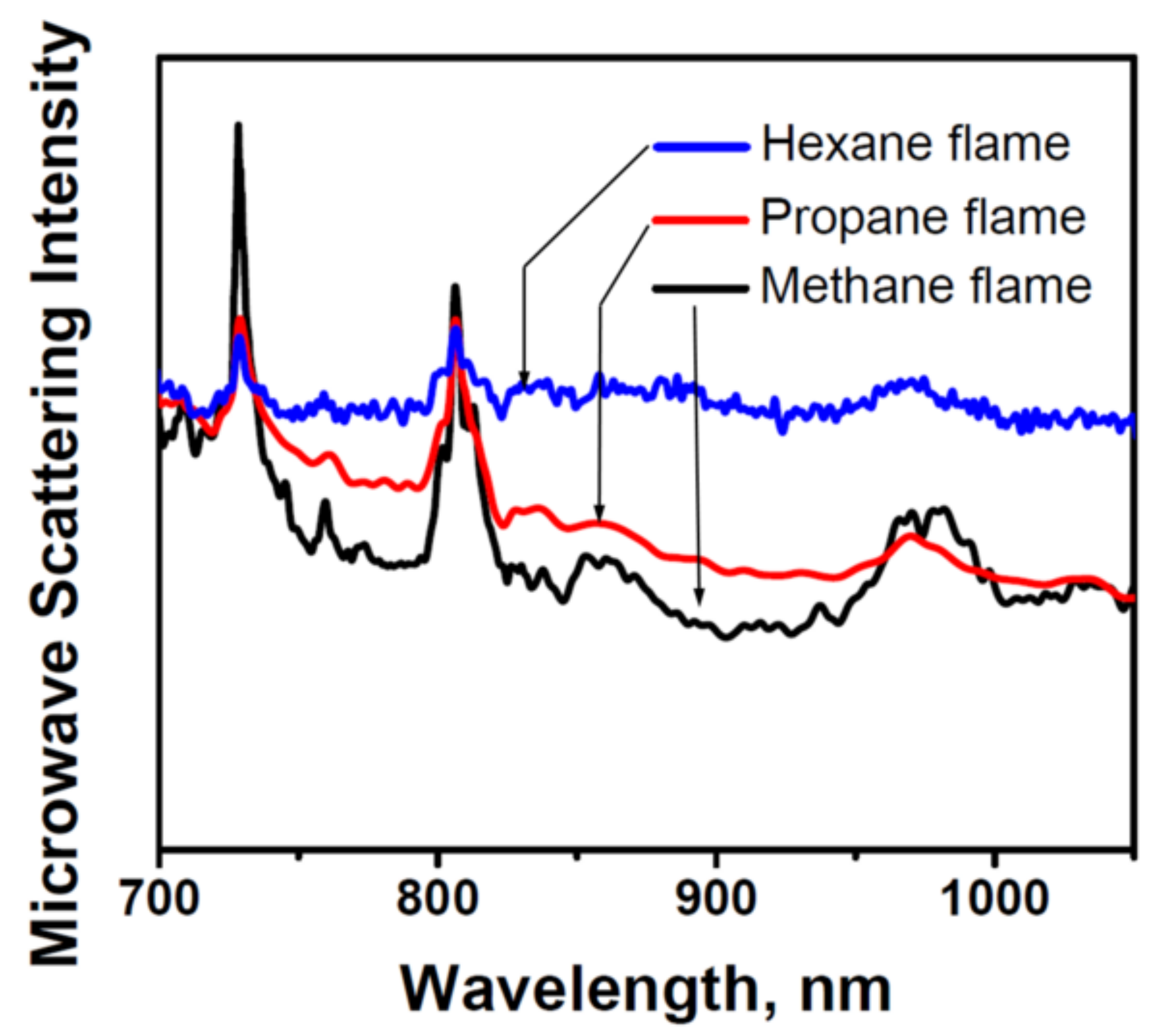




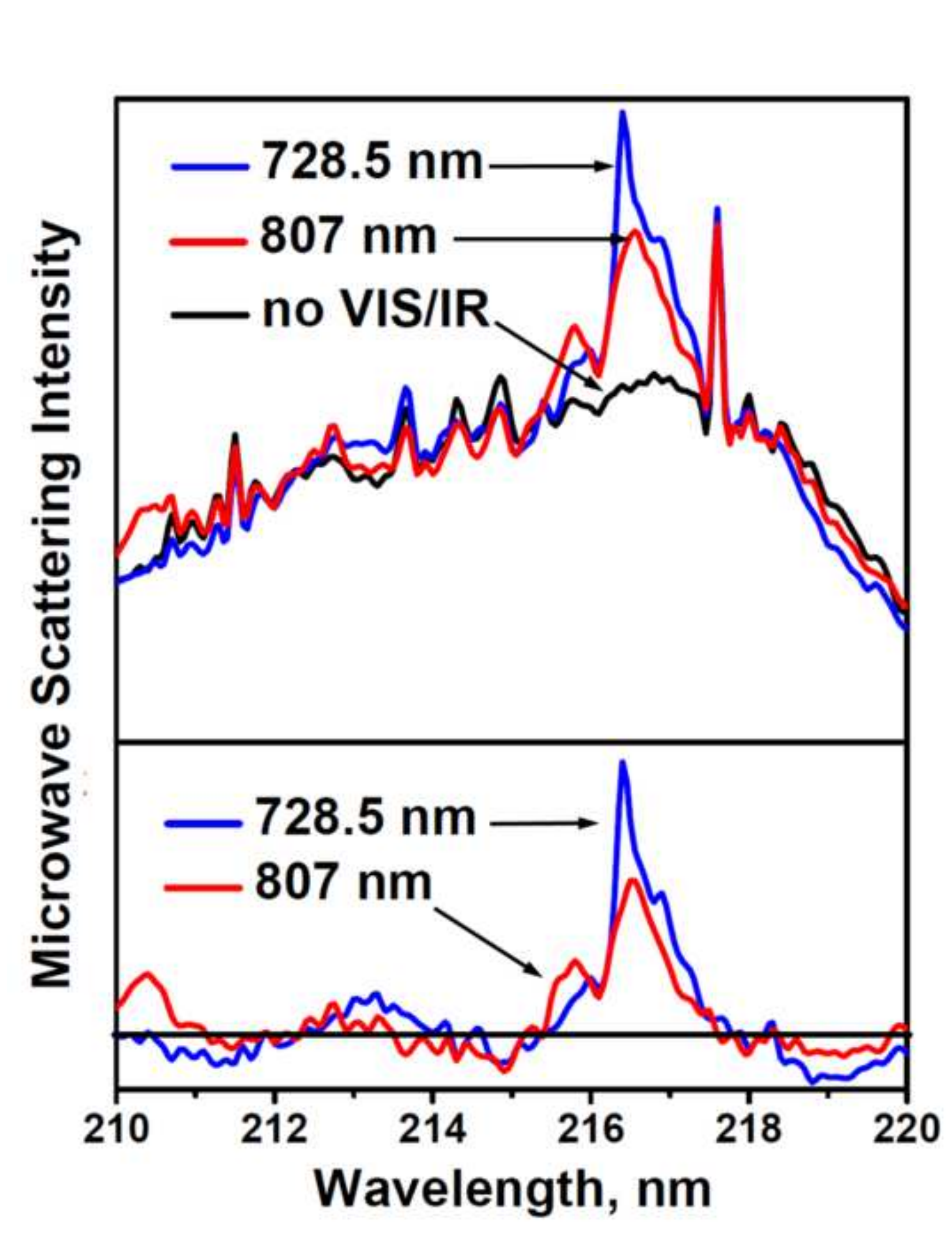

Figure 3
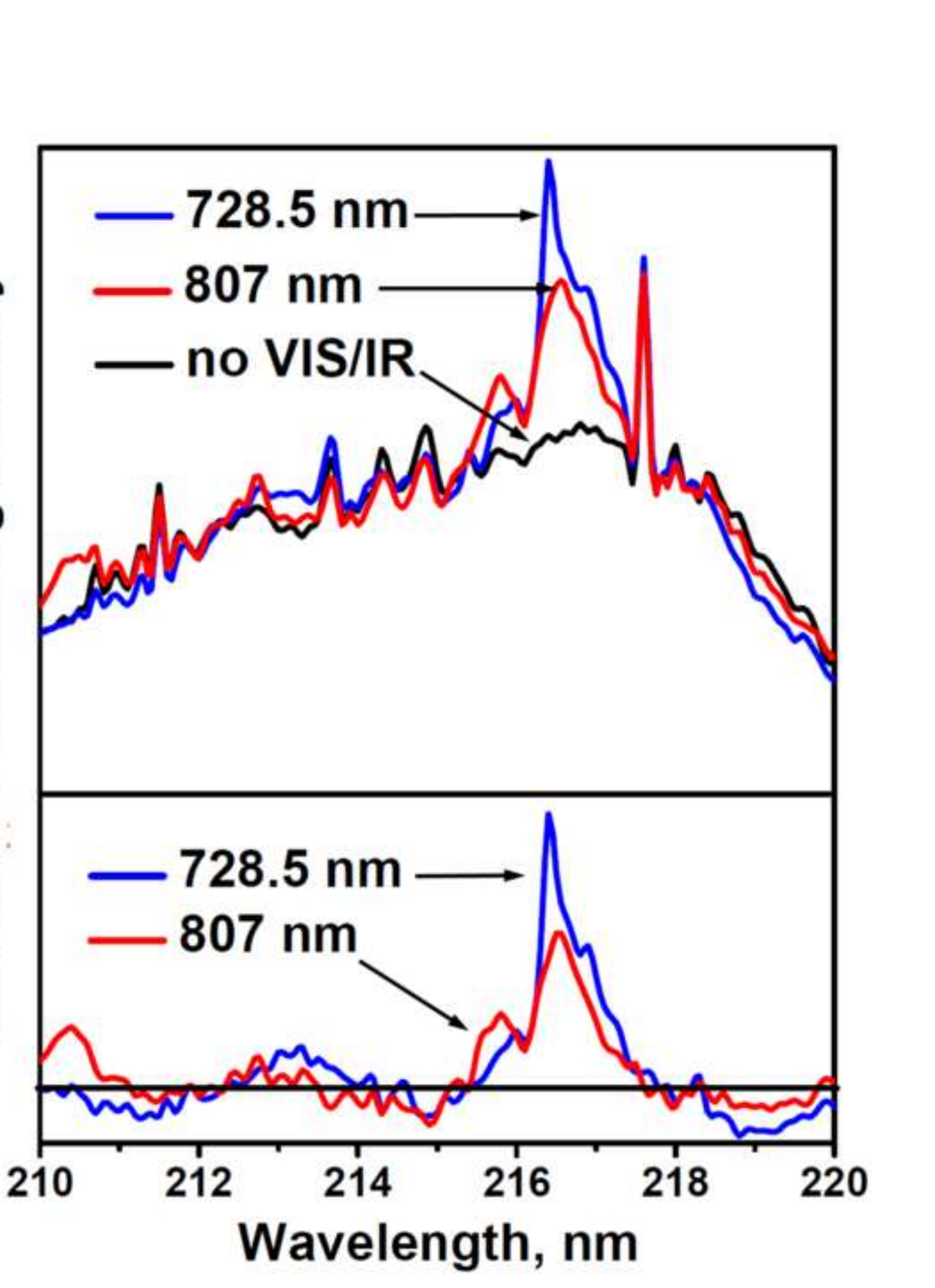

Figure

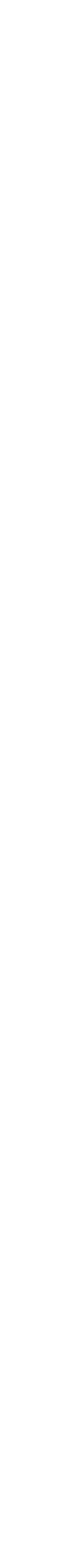
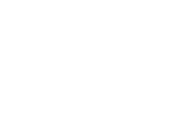
Microwave Scattering

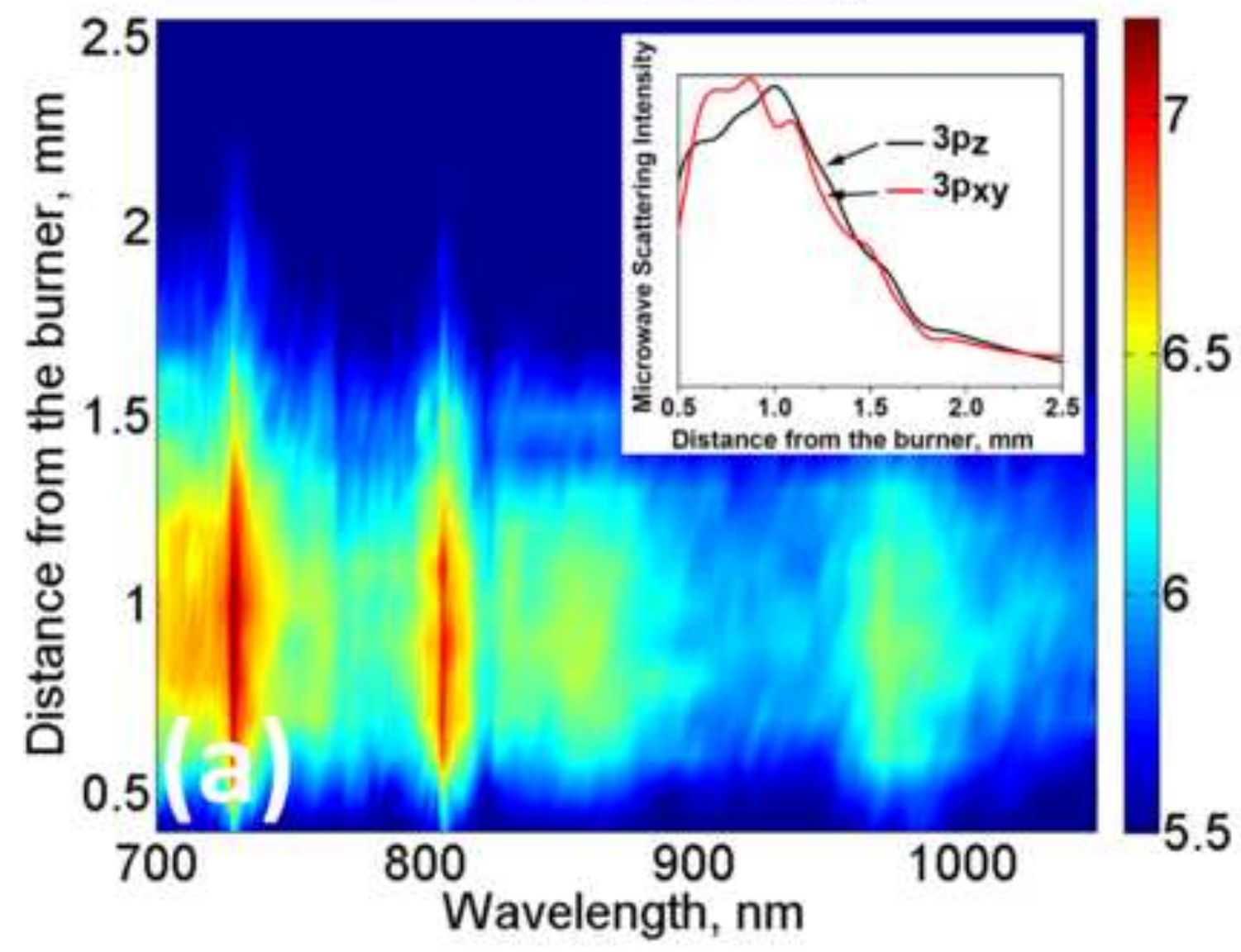

Microwave Scattering

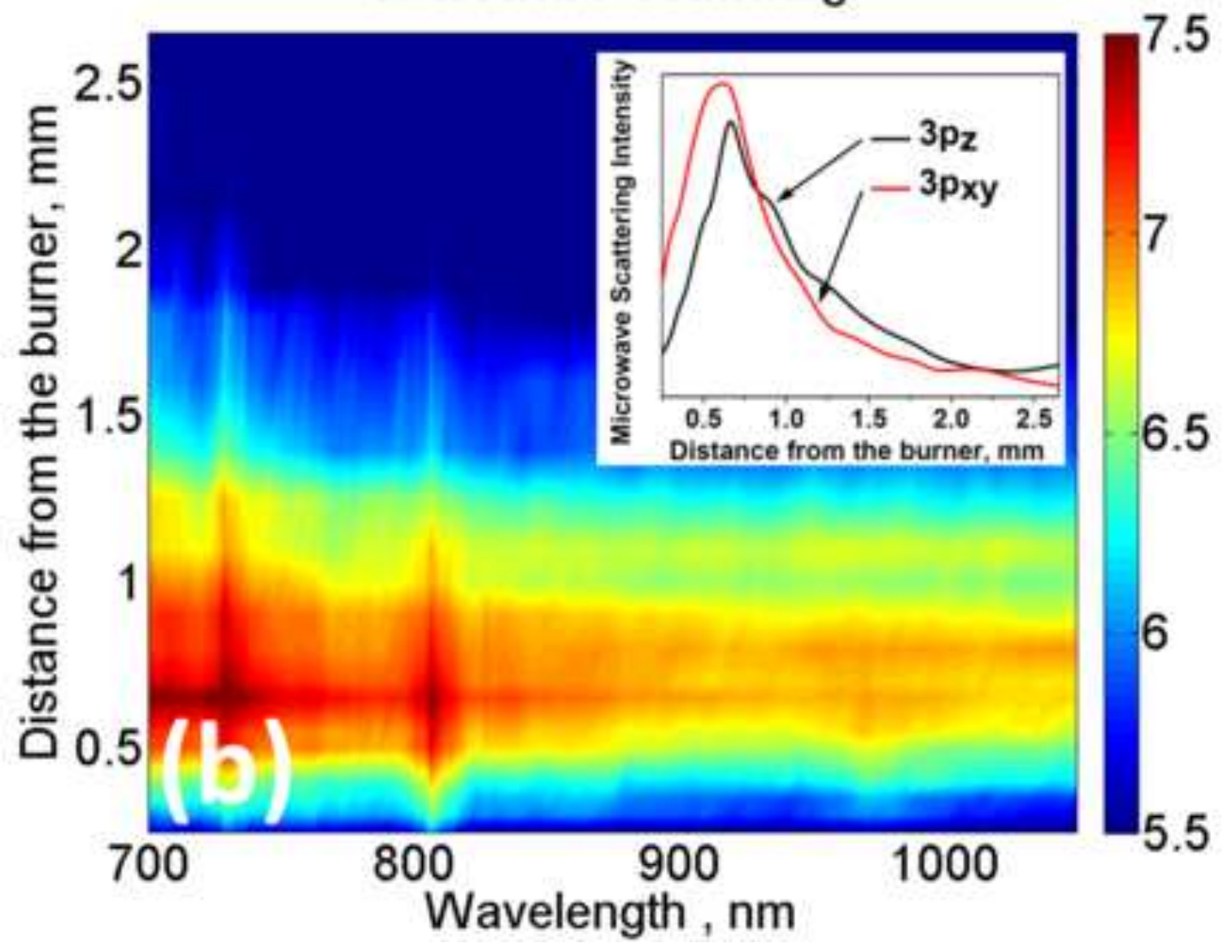

